\title{
Conceptual Design and Preliminarily Structural Analysis of Inflatable Basket for an Asteroid Capturing Satellite
}

\author{
Alessandro Ceruti ${ }^{1,}{ }^{*}-$ Siro Pettenuzzo $^{2}-$ Marco Tuveri $^{3}$ \\ ${ }^{1}$ Industrial Engineering Department - DIN, University of Bologna, Italy \\ 2 School of Engineering and Architecture, University of Bologna, Italy \\ 3 Interdepartmental Centre for Industrial Research in Aeronautics, University of Bologna, Italy
}

\begin{abstract}
This paper presents a conceptual design of a satellite device to be used for capturing asteroids, based on inflatable structures. The mission requirements, the conceptual design, the methodology for the selection of the best solution, and simulations for the preliminarily structural analysis of the device are described within. Several finite element analyses have been carried out in order to find the best strategy to model flexible materials under internal pressure, and the device structure has been changed accordingly in order to obtain the best trade-off between weight and strength. This paper presents the sizing and the weight breakdown of the satellite inflatable components, which are quite complex to model with regards to the structural simulation. The results obtained seem to confirm the feasibility of such a structure and motivates further studies and experimental tests.
\end{abstract}

Keywords: conceptual design, inflatable structure, FEM, MADM, fabric, $\alpha$ damping

Highlights
- $\quad$ Development of a methodology for conceptual design.
- $\quad$ CAD modelling and evaluation of different configurations.
- $\quad$ Preliminary sizing and shape prediction of a soft structure.

\section{O INTRODUCTION}

Several studies have been carried out to study a manned mission to capture asteroids and deflect them into a low earth orbit. This can be useful in developing technologies to deflect asteroids impacting the earth or to study the mineral composition of objects coming from deep space. The feasibility of such a space mission has been demonstrated through the studies by the Keck Institute [1] and [2], by NASA [3] and by several other authors, such as [4]. For instance, the driving idea of Keck's work is to capture an asteroid with a diameter of 7 metres, by launching into orbit a satellite with an inflatable structure to expand in space to envelope the rock. Several vehicles could be useful for launching into orbit such a satellite: e.g. the American Atlas V, the European Ariane V, the Japanese Mitsubishi H-IIA, and the Russian Zenit$2 \mathrm{M}$ are able to carry payloads up to $15,000 \mathrm{~kg}$ of mass, with a maximum diameter ca. $4 \mathrm{~m}$ to $4.5 \mathrm{~m}$. This limit in diameter motivates the use of foldable or inflatable devices for space applications in which a large structure is needed. However, all these conceptual studies require a step into a more detailed design of the mission profile, on the systems to carry and on the structure of such a satellite. Details about the mission trajectory can be studied, following the classic equations of orbital mechanics [5]. However, the design of the satellite configuration and structure needs a more inventive process in which the shape of the inflatable part of the satellite has to be sketched and sized. This paper focuses the attention on this latter aspect, describing the methodology to design and simulate the behaviour of the inflatable part of the satellite through finite element analysis (FEM). A great deal of literature can be found dealing with inflatable structures. The work in [6] presents the development of a mathematical approach to studying the mechanics of plain-woven fabrics, applied to inflated structures; a similar work by Apedo et al. [7] focuses the attention on the vibration modes of inflatable beams built with orthotropic woven fabric. Applications of inflated structures range from space and aeronautical [8] applications (e.g. inflated wings) to civil engineering (bridges with Tensairity technology) to food processing. In this latter topic, it is worth citing the work by [9] in which the design of inflatable open-ocean-aquaculture cages is carried out in a rigorous way, by applying the leading edge technologies related to the numerical modelling and simulation of inflatable structures. Focusing the attention on the modelling of inflatable structure for space applications, for instance, another paper [10] compares two off-the-shelf simulation codes (LSDYNA and ADAMS) in predicting the behaviour of inflatable space structures. An example of the 
design, FEM analysis and testing of an inflatable space structure is presented in [11], in which the development of an inflatable re-entry vehicle based on a cone-shaped configuration made by a series of inflated rings is described in theoretical, numerical and experimental aspects. The paper [12] shows a good agreement between simulation results obtained with the LS-DYNA code and the experimental tests on the airbag structure of the Orion crew module; the work by [13] also describes a review of the methods for the structural analysis of airbags, focusing the attention on the problem of reducing the effects of the landing on the Mars surface, topic faced by [14] as well. The use of inflatable structures and methods for its structural analysis are also described with reference to expandable lunar habitats [15], and to antennas for small Cubesat satellites [16]. The papers [17] and [18] represent some of the most recent studies on the structural analysis of inflatable structures for space applications. The concepts and experiences presented by these authors in the design and structural modelling of inflatable structures have been taken into account in the work described in this paper.

The structure of the paper is as follows: the first section describes the design requirements of the device, the second presents the deployment of the design phase and shows some sketches of the proposed configurations. After the selection of the most promising inflatable shape, the third section describes the methodologies explored to carry on the structural analysis and to set the thickness of this structure. A final section includes a conclusion and some comments on the most significant results obtained in this work.

\section{DESIGN REQUIREMENTS}

The aim of this work is to present a possible methodology to size and test the structure of the inflatable section of a satellite conceived to capture an asteroid. Following the studies by [1] and [2], attention was focused on the design of a structure to capture an asteroid of a free form shape, with a main length of 7 $\mathrm{m}$, and a mass of about $500,000 \mathrm{~kg}$; the real asteroid mass could range between $250,000 \mathrm{~kg}$ and $1,000,000$ $\mathrm{kg}$, depending on its composition [1]. A conceptual layout of the satellite components is presented in Fig. 1. The satellite core contains the inflatable structure during launch, compressed gas or chemical powders for inflation, electronics, batteries, thrusters, and propellant. A Stewart platform between the inflatable basket and the satellite core could be used to align the asteroid centre of gravity with the axis of symmetry of the satellite itself to reduce eccentric effects during the thrust phase.

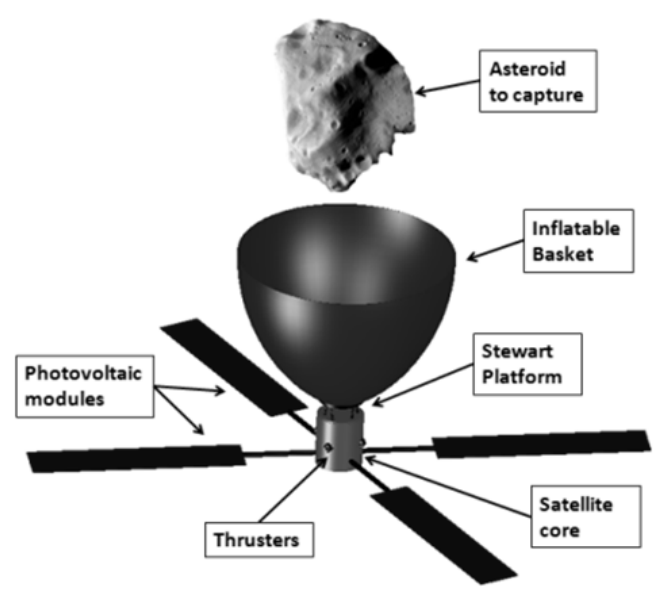

Fig. 1. Conceptual layout of the device

Fig. 2 presents the main dimensions of the satellite core, based on what is suggested by literature [1], and an average sizing of the basket.

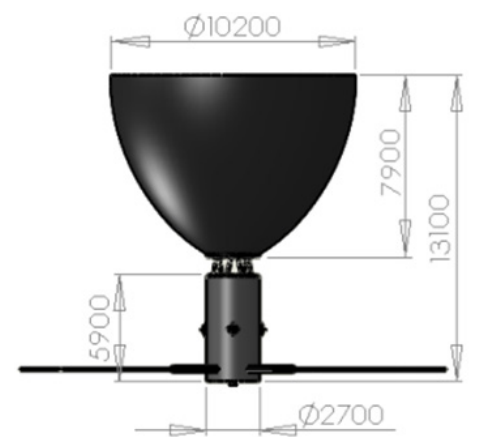

Fig. 2. Average dimensions of the satellite

The satellite should approach the asteroid, envelope it with the inflatable structure, and finally secure and block it inside the basket to change its trajectory: a complex docking algorithm [1] controlling the thrusters will be used to reduce the impact forces between the asteroid and the basket. The selected configuration is based upon a main inflatable structure shaped like a cone, with internal appendices that could be further pressurized up to touching the external surface of the asteroid; in such a manner, the contact force between the appendices and the asteroid assures a friction force blocking the rock into the satellite. A detailed design of the basket, starting from the abovedescribed requirements is presented in the following. The materials for the basket have been initially selected according to the literature: a sandwich of fabrics in Vectran ${ }^{\circledR}$ and $\operatorname{Kevlar}^{\circledR}$ seems to be the most 
effective solution available. The properties of these materials are included in the following Table 1. Thin thermal insulation layers in Aerogel ${ }^{\circledR}$ and airtight in Kapton $^{\circledR}$ can also be added.

Table 1. Material properties

\begin{tabular}{lcc}
\hline \multicolumn{1}{c}{ Name } & Vectran & Kevlar \\
\hline Elastic module $\left[\mathrm{N} / \mathrm{mm}^{2}\right]$ & 103000 & 127500 \\
\hline Tensile strength $\left[\mathrm{N} / \mathrm{mm}^{2}\right]$ & 2900 & 3600 \\
\hline Poisson's Coefficient [-] & 0.3 & 0.34 \\
\hline Density $\left[\mathrm{kg} / \mathrm{m}^{3}\right]$ & 1400 & 1400 \\
\hline
\end{tabular}

\section{CONCEPTUAL DESIGN AND METHODS FOR BEST SOLUTION DEFINITION}

After the clarification of the design scope, the stateof-the art analysis, and the definition of design requirements, a set of conceptual sketches of possible configurations of the basket has been carried out. Finally, these shapes have been evaluated in order to select the most suitable one and to proceed with its structural sizing.

\subsection{CAD Modelling of Configurations}

Some conceptual configurations have been initially sketched by hand, and in the following they have been modelled within a computer-aided design (CAD) software. In the first configuration C1 (Fig. 3), named "Bubble", a thin basket with a conical shape is added by a set of spheres whose function is to soften the impact of the asteroid and further to block it inside the device.

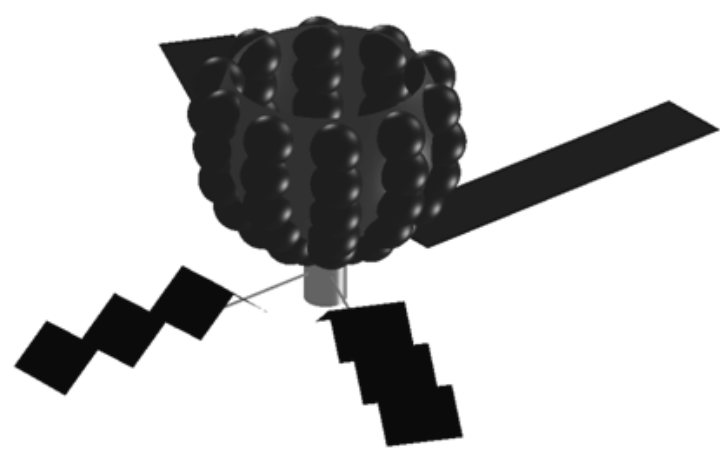

Fig. 3. Conceptual CAD model of the "Bubble" configuration

The second configuration C2 (Fig. 4), named "Reticular", is based on a reticular structure obtained through wires inclined $45^{\circ}$ respect to the axis of the cone. They stiffen a series of 12 inflatable strips, each one equipped with 7 semi-spheres.

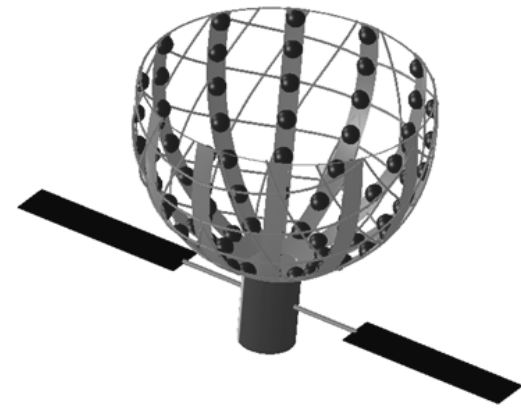

Fig. 4. Conceptual CAD model of the "Reticular" configuration

The third configuration C3 (Fig. 5), called "Spiral", is made by an inflatable spiral tube dressing all the cone, from the base to the top. Along the tube, a series of inflatable balls helps in damping the impact of the asteroid and the satellite, blocking it inside. A thin layer of fabric is connected to the tube to maintain the conical shape.

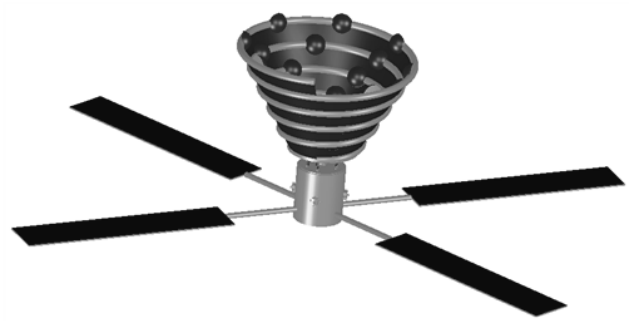

Fig. 5. Conceptual CAD model of the "Spiral" configuration

The fourth proposed configuration $\mathrm{C} 4$, called "Spheres \& Cones", is similar to the first one, since it is based upon an inflatable cone; the main difference is that it is equipped with inflatable semi-spheres and large cones whose function is to block the asteroid inside.

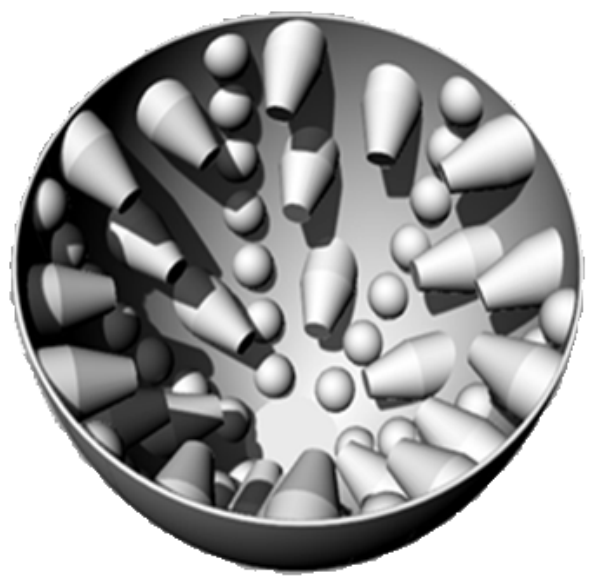

Fig. 6. Conceptual CAD model of the "Spheres \& Cones" configuration 


\subsection{MADM Approach to Select the Best Configuration}

A Multi Attribute Decision Making (MADM) approach [19] and [20] has been followed to evaluate the most effective configuration. It will be sized and verified with FEM analysis in the following of the paper. The MADM's first step is to interview experts on the topic asking for the attributes they consider critical for a product. After the interview, a series of attributes are collected; similar concepts are usually condensed into a single attribute to reduce their number $N$ to a manageable value.

Table 2. MADM matrix of attributes relative importance (R.I.)

\begin{tabular}{cccccc}
\hline & $A_{1}$ & $A_{i}$ & $A_{N}$ & Sum & R.I. \\
\hline$A_{1}$ & $S_{1,1}$ & $S_{1, i}$ & $S_{1, N}$ & $S_{1}=\sum_{i=1}^{N} S_{1, i}$ & $R I_{1}=\frac{S_{1}}{S S}$ \\
\hline$A_{j}$ & $S_{j, 1}$ & $S_{j, i}$ & $S_{j, N}$ & $S_{j}=\sum_{i=1}^{N} S_{j, i}$ & $R I_{j}=\frac{S_{j}}{S S}$ \\
\hline$A_{N}$ & $S_{N, 1}$ & $S_{N, i}$ & $S_{N, N}$ & $S_{N}=\sum_{i=1}^{N} S_{N, i}$ & $R I_{N}=\frac{S_{N}}{S S}$ \\
\hline & & & $S S=\sum_{j=1}^{N} S_{j}$ \\
\end{tabular}

In the following, these attributes $\left(A_{1}, A_{2}, A_{i}, \ldots, A_{N}\right)$ are listed in row and column in a matrix, as Table 2 shows. A score of 0 is assigned to the intersection between line and column if the attribute in the row $\left(A_{j}\right)$ is less important than that in the column $\left(A_{i}\right)$, 1 if the comparison is a draw, and 2 in the opposite case. Finally, the scores are summed row by row, and the result is assigned to the attribute in the row of the matrix. By summing all the scores of the single attributes and dividing the single attribute score $\left(S_{j}\right)$ by this sum (SS), the relative importance of the attribute $\left(R I_{j}\right)$ is found.

Afterward, each configuration is evaluated by assigning a score $(V)$ from 1 to 5 to the satisfaction of each single attribute.

The final configuration suitability $(F S)$ is found by multiplying the score times the relative importance (R.I.) of the attribute and summing all these terms (see Table 3). The following methodology has been applied to the case study: after an interview with several experts in the field of space structures and missions, seven attributes for the basket have been detected as significant for the selection of the configurations, as can be seen in Table 4. The attributes (from $A_{1}$ to $A_{7}$ ) have been evaluated using the matrix of Table 2, and relative importance indexes have been obtained.

Table 3. MADM evaluation of configurations

\begin{tabular}{ccccc}
\hline & $A_{1}$ & $A_{i}$ & $A_{N}$ & Sum \\
\hline Conf. \#1 & $V_{1,1}$ & $V_{1, i}$ & $V_{1, N}$ & $F S_{1}=\sum_{i=1}^{N} R I_{i} \times V_{1, i}$ \\
\hline Conf. \#j & $V_{j, 1}$ & $V_{j, i}$ & $V_{j, N}$ & $F S_{j}=\sum_{i=1}^{N} R I_{i} \times V_{j, i}$ \\
\hline Conf. \#M & $V_{M, 1}$ & $V_{M, i}$ & $V_{M, N}$ & $F S_{N}=\sum_{i=1}^{N} R I_{i} \times V_{M, i}$ \\
\hline
\end{tabular}

Table 4. List of attributes

\begin{tabular}{cl}
\hline$\#$ & Description \\
\hline$A_{1}$ & Symmetry of the basket \\
\hline$A_{2}$ & Stability of the basket shape under external loads \\
\hline$A_{3}$ & Capability of blocking free form shaped asteroids \\
\hline$A_{4}$ & Folded volume (before expansion) \\
\hline$A_{5}$ & Easiness of inflation \\
\hline$A_{6}$ & Weight and surface \\
\hline$A_{7}$ & Retaining of debris eventually detached by asteroid \\
\hline
\end{tabular}

Finally, using the matrix described in Table 3 , all the four configurations $\left(C_{1}\right.$ to $\left.C_{4}\right)$ have been scored: the "Spheres \& Cones" configuration obtained the best score, as Table 5 shows.

Table 5. Relative importance of attributes and final suitability of configurations

\begin{tabular}{|c|c|c|c|c|}
\hline & R.I. [\%] & Conf. \# & Configuration icon & FS [-] \\
\hline$A_{1}$ & 9.8 & \multirow{2}{*}{$C_{1}$} & & \multirow{2}{*}{3.94} \\
\hline$A_{2}$ & 21.6 & & & \\
\hline$A_{3}$ & 19.6 & \multirow{2}{*}{$C_{2}$} & & \multirow{2}{*}{3.47} \\
\hline$A_{4}$ & 11.8 & & & \\
\hline$A_{5}$ & 11.8 & \multirow{2}{*}{$C_{3}$} & & \multirow{2}{*}{3.1} \\
\hline$A_{6}$ & 9.8 & & & \\
\hline$A_{7}$ & 15.7 & \multirow{2}{*}{\multicolumn{2}{|c|}{$C_{4}$}} & \multirow[b]{2}{*}{4.16} \\
\hline & & & & \\
\hline
\end{tabular}




\subsection{Architecture and Layout of the Most Promising Solution}

Following this evaluation phase, the configuration $C_{4}$ has been selected for further analysis and it has been modelled with a higher detail. The configuration $C_{1}$ achieved a good score, but the absence of cones would reduce the capability to block the asteroid; the configuration $C_{2}$ presents the drawback of releasing eventual debris detached from the asteroid in the outer space with the risk of impacts on the satellite structure or solar panels. Finally, the configuration $C_{3}$ is highly asymmetric and seems to be problematic in the case of heavy impacts.

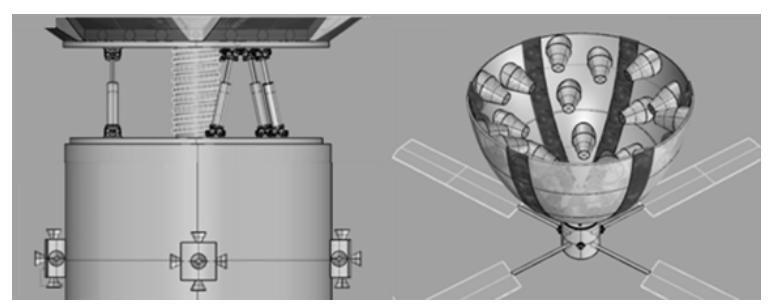

Fig. 7. Satellite details (bubbles in dark strips)

The dimensions of the semi-spheres have been preliminarily set equal to $1 \mathrm{~m}$, while the cones present a height of $1.5 \mathrm{~m}$, a base and top diameter of $1 \mathrm{~m}$ and $0.6 \mathrm{~m}$ respectively.

\section{METHODOLOGIES FOR INFLATABLE STRUCTURAL ANALYSIS}

Several strategies have been evaluated to size the structure and test its strength. The system, which is fully inflatable, is composed of a fabric made by a dense network of multilayer fibres of Vectran and Kevlar. The Nastran/Patran environment and the explicit solver of the Dytran module have been used to obtain the results presented in the following of the paper.

\subsection{Load Evaluations and Rationale}

The load evaluation on the structure of the satellite basket during a mission of asteroid capturing is a very complex task. Following the study [21] describing criticalities on trajectories for capturing missions, there are several issues to consider: the shape of the asteroid which can be spherical or more likely oblong, its inertia matrix and rotational speed, its solidity, possible impacts during docking, and the change in centripetal acceleration due to the rotational speed of the satellite during the engagement phases.
Due to all these uncertainties, it is extremely difficult to simulate a real scenario of docking. The structure we have conceived has a conical part, which should keep its shape during the capturing, and cones and spheres whose pressure depends on the gripping force needed to block the asteroid.

The amount of this force depends on the solidity of the rock, which can be affected by its rotational speed, the friction coefficient, which changes with the external shape and material of the rock, and the inertial loads due to the thrusters, which are usually very low. According to the study [1] and [21], it can take months or years to de-spin or de-tumble asteroids. Due to this framework of uncertainties, what we present in this paper is a comparative study of several structural implementations of the basket structure to evaluate the withstanding of the internal pressure and the final shape after inflation: a series of analysis has been carried out by simulating the stresses and deformations following the inflation of the basket with an increasing pressure; the main inflatable structure, consisting of two internal and external conical surfaces connected by ribs, has been constrained at the base to simulate the connection with the satellite. The structure has been tested with an internal proof pressure of 0.5 bar, i.e. a value similar to what presented in [12] where a pressure discharge test from 0.55 bar up to 0 bar in a time of $0.12 \mathrm{~s}$ is described for an inflatable space structure. As already stated, the inflation pressure of the spheres and cones is variable depending on the mission profile and asteroid features; however, some simulations for this appendices with the same pressure used for the main inflatable structure, which is considered a worst case study, have also been carried out.

\subsection{Analysis with Equivalent Isotropic Material and 12 Vertical Ribs}

In the first set of analyses, the structure has been simplified for FEM analysis by removing the semispheres and cones, which will be analysed later. Twelve vertical ribs (one every $30^{\circ}$ ) have been modelled to stiffen the structure, which is essentially composed of two parallel inflated conical surfaces (see Fig. 7). The ribs are flexible plain strips of the same material used for the envelope spanning between the internal and external conical surfaces. The mesh has been obtained panelling the surfaces of the basket and the internal ribs with triangular shell elements. 

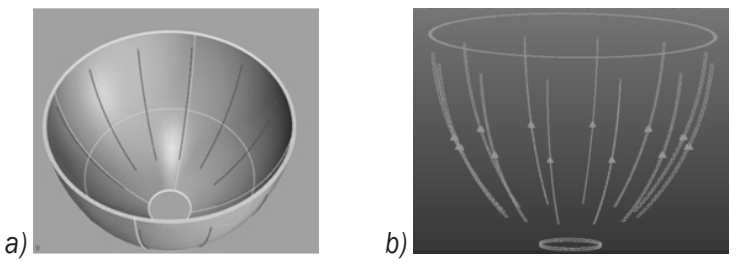

Fig. 8. Structure with 12 vertical ribs; a) $C A D$ and b) meshing of the ribs

At first, as suggested by literature, [12], a homogeneous isotropic material was used instead of a fabric modelling. The material model (Young modulus and Poisson modulus) adopted in this first set of tests, has been computed with the formula:

$$
\bar{x}=\sum_{i=1}^{n} \frac{x_{i} \cdot s_{i}}{s_{t o t}},
$$

where $x_{i}$ represents a generic property of the laminated; $s_{i}$ is the thickness of the material layer, $s_{t o t}$ is the total thickness of the stacking. In these tests, the fabric thickness is set equal to $2 \mathrm{~mm}$, with a mixture 1:1 of Kevlar and Vectran in thickness. From this nonlinear analysis, a high deformation is noticed, since the structure withstands the pressure loads poorly: a maximum displacement of $1.7 \mathrm{~m}$ is detected, with a maximum stress of $1250 \mathrm{MPa}$ (see Fig. 9), and some peaks up to $2380 \mathrm{MPa}$, close to the breaking stress of the material.
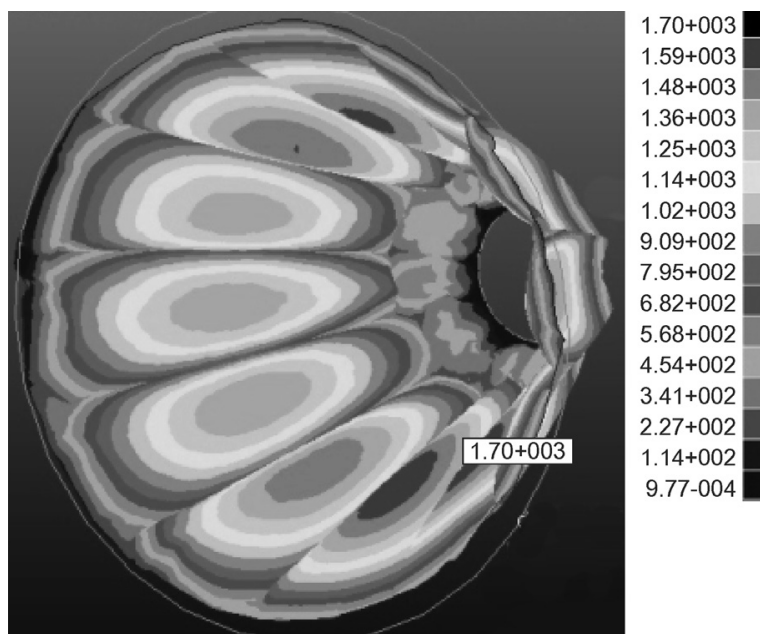

Fig. 9. FEM analysis of the internal envelope only with 12 vertical ribs: displacements in $\mathrm{mm}$

This result is due to the modelling of the fabric with a homogenous isotropic material: it withstands both compression and traction loads, so that the element used for these simulations increases the stress due to the compression of the fabric. Following the physics of the problem, the compression strength of the fabric is well below the tensile one, so that it does not present resistance to the compression. In this first set of tests with equivalent isotropic material, a Von Mises breaking criteria, which keeps into account both compression and tensile stresses, has been used:

$$
\sigma_{v}=\sqrt{\begin{array}{l}
\frac{1}{2}\left[\left(\sigma_{11}-\sigma_{22}\right)^{2}+\left(\sigma_{22}-\sigma_{33}\right)^{2}+\right. \\
\left.\left(\sigma_{33}-\sigma_{11}\right)^{2}+6\left(\sigma_{12}^{2}+\sigma_{23}^{2}+\sigma_{31}^{2}\right)\right]
\end{array}},
$$

where $\sigma_{v}$ is the Von Mises stress, and $\sigma_{i j}$ are the elements of the $3 \times 3$ stress matrix. With a thickness of the structure of $2 \mathrm{~mm}$, the mass of the basket is $1188 \mathrm{~kg}$ (38 kg for the ribs, $579 \mathrm{~kg}$ for the external structure, $560 \mathrm{~kg}$ for the internal structure, $11 \mathrm{~kg}$ for the top and down closing edge surfaces).

\subsection{Equivalent Isotropic Material and 24 Vertical Ribs}

Following the goal of reducing the displacement of the structure under internal pressure, a second structural layout has been implemented with 24 vertical ribs, one every $15^{\circ}$, as Fig. 10 shows, and the same material properties, loads and constraints of the previous set of FEM analyses.
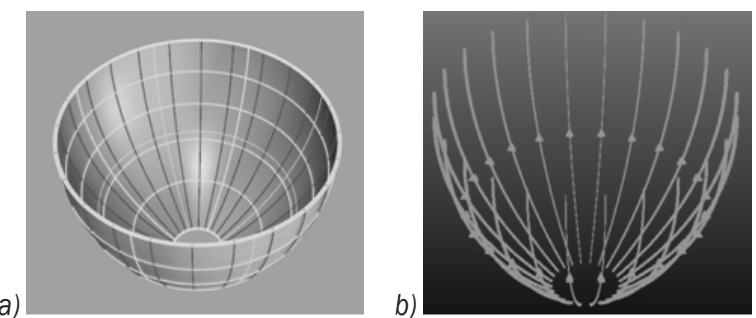

Fig. 10. Structure with 24 vertical ribs; a) $C A D$ and b)meshing of the ribs

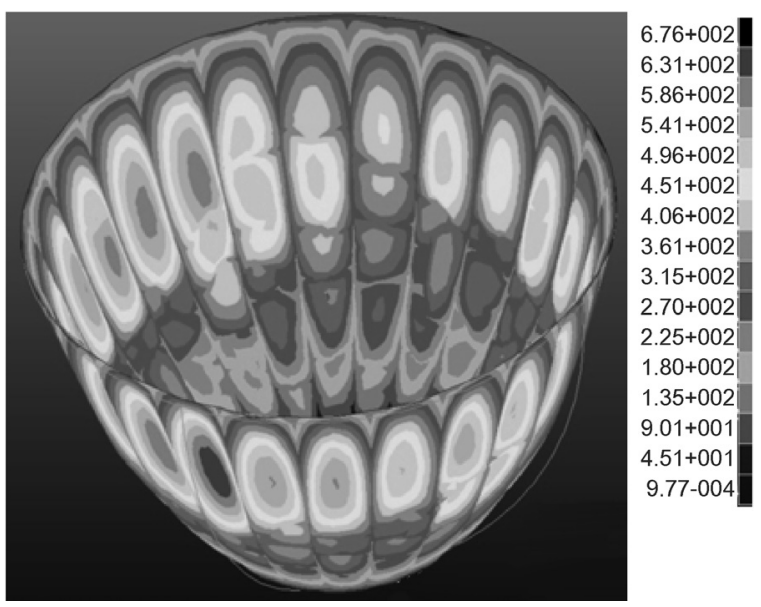

Fig. 11. FEM analysis with 24 vertical ribs: displacements in $\mathrm{mm}$ 
By introducing 24 ribs, the structure displacement is reduced to $0.68 \mathrm{~m}$ : a peak stress of $2090 \mathrm{MPa}$ in the basket internal part is observed. Similarly to the previous analysis, in this case the trend of the displacement of a test point of the structure also presents an oscillation (see Fig. 12), showing an instability in the solution and a partial convergence of the results. However, better results with respect to the 12 ribs case has been achieved.

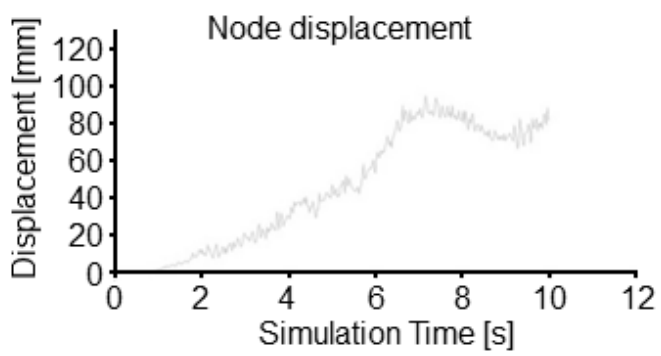

Fig. 12. Convergence of the displacement of a node as a function of simulation time

\subsection{Basket with 9 Horizontal Ribs}

The need for a reduction in structure weight has led to the testing of other configurations. As Fig. 13 shows, the basket has been stiffened with 9 horizontal parallel ribs, perpendicular to the basket vertical axis of symmetry.
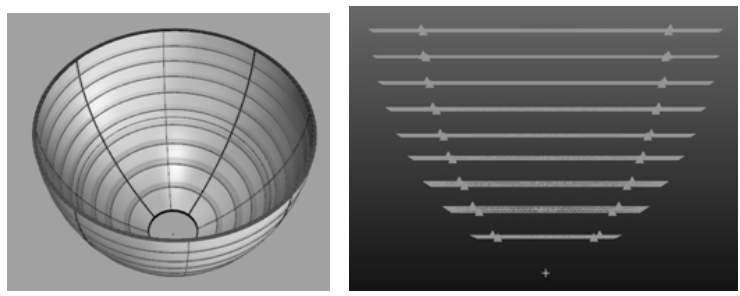

Fig. 13. Structure with 9 horizontal ribs: CAD and meshing of the ribs

The FEM analysis (Fig. 14) showed a maximum deformation of $292 \mathrm{~mm}$, and a maximum stress of $1300 \mathrm{MPa}$. However, problems in solution stability and convergence remain; the structure is unstable under the pressure load, and an equilibrium is not found even for simulation times well beyond the end of the pressurization process.

\subsection{Damping Coefficient and Modal Analysis}

Following literature suggestions [22] related to analysis of non-linear structures [23], two methods can be followed in non-linear analysis requiring damping: the $\alpha$ damping, and the global C-matrix or system damping.

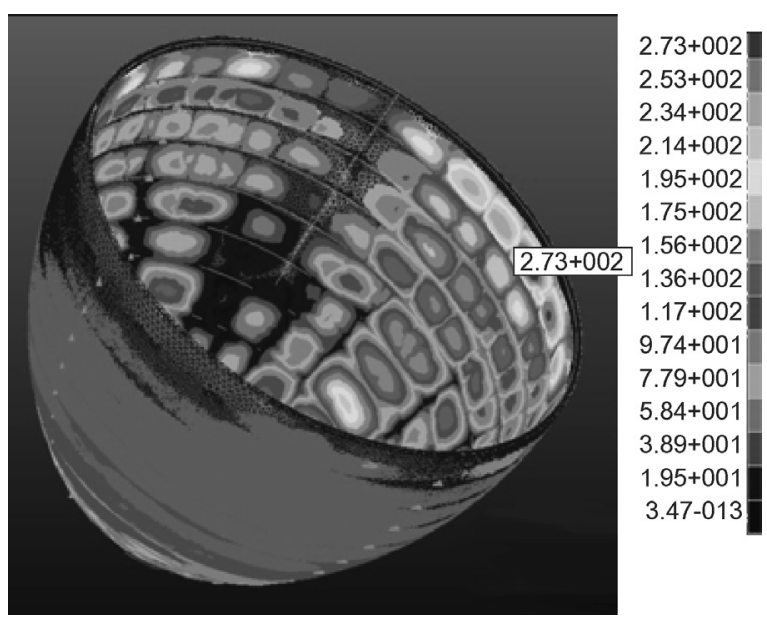

Fig. 14. FEM analysis of the structure with 9 horizontal ribs: displacements in $\mathrm{mm}$

The C-matrix approach is based upon a mass spring system whose motion equation is:

$$
M a^{n}+C v^{n}+F_{\text {int }}^{n}=F_{\text {ext }}^{n} .
$$

The $M$ matrix represents the masses matrix, $F_{\text {int }}$ the elastic internal forces due to springs' deformation, $F_{\text {ext }}$ the external forces, and $C$ is the damping matrix (diagonal).

In the $\alpha$ damping, selected in this study, the solver uses a single damping parameter, which is introduced in the central differences solution scheme. The equation is:

$$
v^{n+1 / 2}=v^{n-1 / 2}(1-\alpha)+a^{n} \Delta t,
$$

where $v$ represents the speed of the single node, $a$ its acceleration, $\Delta t$ the integration step, while $\alpha$ is the dynamic relaxing parameter. Literature, [19], suggests that the $\alpha$ coefficient should be equal to $5 / 3$ times the critical damping of the whole system, or, in other words, $5 / 3$ times the system natural frequency multiplied by the integration step $\Delta t$.

For instance, the natural frequency $\left(f_{i}\right)$ of a singledegree-of-freedom system can be computed with:

$$
f_{i}=\frac{\omega_{i}}{2 \pi}=\frac{1}{2 \pi} \sqrt{\frac{k_{i}}{m_{i}}} .
$$

When a complex structure with many degrees of freedom is analysed, usually the lowest natural frequency $\left(f_{1}\right)$ is considered. 
A modal analysis of the configurations with vertical and horizontal ribs has been carried out, aiming to find the natural frequencies of these structures (see Fig. 15).
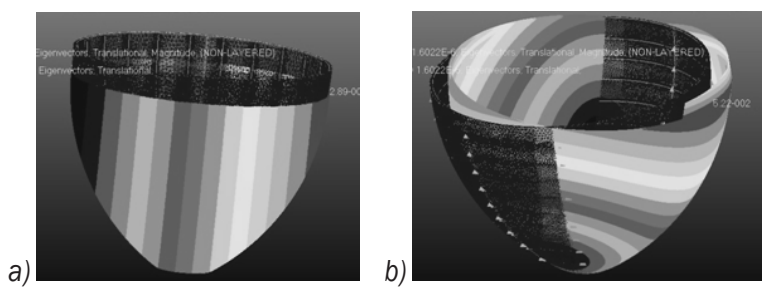

Fig. 15. Basket deformations for the first natural frequency with a) vertical and b) horizontal ribs solutions

For the structure with 24 vertical and 9 horizontal ribs, the first three resulting frequencies were found respectively: 1.465E-6, 2.28E-4, 3.945E-4, 1.60E-6, $1.5685 \mathrm{E}-6$ and $1.28 \mathrm{E}-6$.

It was possible to compute the $\alpha$ damping coefficient:

$$
\alpha=\frac{5}{3} f_{\text {nat }} \Delta t
$$

The $\Delta t$ used in the above equation has been set using the equation:

$$
\Delta t=\frac{t h}{\sqrt{E / \rho}}
$$

It represents the time a sound wave takes to pass through the thinnest element of the structure, represented by the fabric thickness. $E$ is the elastic module of the material, $\rho$ its density and $t h$ stands for the fabric thickness. Table 6 lists the computed values for the first natural frequencies $\left(f_{1}\right)$ and $\alpha$ damping coefficients.

Table 6. Damping coefficients

\begin{tabular}{cccc}
\hline Structure & $f_{1}$ & $\Delta t$ & $\alpha$ \\
\hline Vertical ribs (24) & $1.465 \mathrm{E}-6$ & $2.90 \mathrm{E}-5$ & $7.1 \mathrm{E}-11$ \\
\hline Horizontal ribs (9) & $1.602 \mathrm{E}-6$ & $3.971 \mathrm{E}-5$ & $1.06 \mathrm{E}-10$ \\
\hline
\end{tabular}

Some convergence tests have been carried out in order to check the structural behaviour of the basket during non-linear analysis. The theoretical value of the $\alpha$ parameter of relaxation (equal to 7.1E-11) does not provide solution convergence, so a systematic analysis was carried out to find a value providing the nodes displacement convergence. Five values of the $\alpha$ parameter were tested: 7.1E-11, 1E-5, 1E-4, 1E-3 and $1 \mathrm{E}-2$.

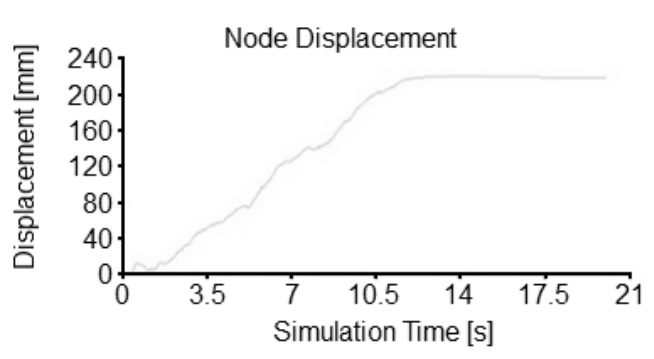

a)

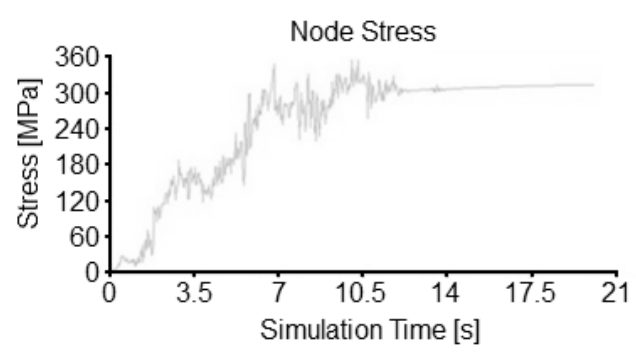

Fig. 16. Example of a) node displacement, and b) stress convergence with $\alpha$ damping

The best results have been obtained with $\alpha=1 \mathrm{E}-4$ : the value suggested by literature seems to be too small, while the $1 \mathrm{E}-3,1 \mathrm{E}-2$ values provide an over-damped response of the structure. In this case, the analysis presents a stable value for nodes displacement and stress, as Fig. 16 shows. Nevertheless, as Fig. 17 shows, there are quite large zones where the compression stresses are high. As stated before, this is not a real effect since the fabric opposes a small resistance to the compression; according to the real material behaviour, the fabric deforms up to equilibrate stable configuration, keeping low compression stresses.

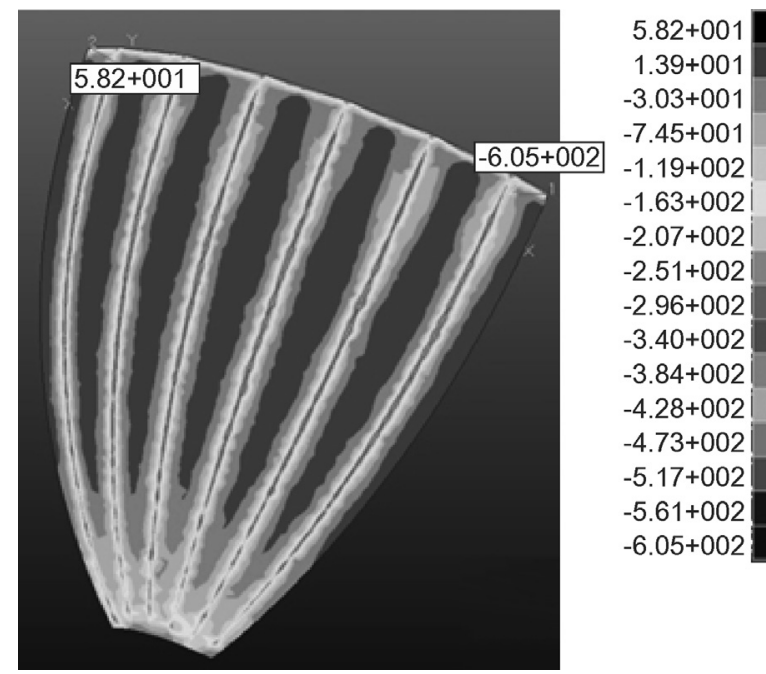

Fig. 17. Vertical ribs configuration FEM analysis with damping coefficient: compression zones, Stresses in $\mathrm{MPa}$ 


\subsection{Analysis with Fabric Model}

The previously presented analysis showed that good structural behaviour can be obtained with the horizontal ribs configuration. It was also observed that the damping coefficient is fundamental in order to achieve the solution stability. However, to better simulate the compression behaviour of the material, a final improvement in the model should be carried out. The compression weakness of the fabric should be in fact considered to obtain a solution closer to the real physic of the problem (in [12] the compressing stress elimination setting of LS-DYNA was selected).

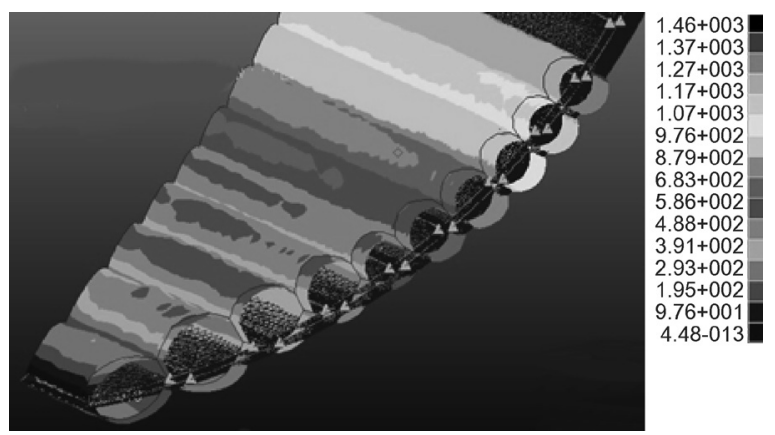

Fig. 18. Horizontal ribs model FEM analysis with damping and fabric element: displacements in $\mathrm{mm}$

In a new set of tests, the structure with horizontal ribs was modelled with an orthotropic fabric material model, and a membrane element of Patran/Nastran, with a $\pm 90^{\circ}$ stacking: the fabric thickness was set equal to $2 \mathrm{~mm}$, with an equal mixture of Kevlar and Vectran sheets and a symmetric lay-up with regards to the mid-plane.

The FEM analysis (see Fig. 18) showed behaviour consistent with the physics of the problem, and a maximum displacement of $1.46 \mathrm{~m}$, with a tensile stress of $98.2 \mathrm{~N} / \mathrm{mm}^{2}$. The behaviour of displacements and stresses (as a function of time and inflation process) presents a very smooth trend up to the convergence to a final value. Thanks to the low values of stress in the structure, the thickness of the fabric was reduced to $0.5 \mathrm{~mm}$, and further analyses following the Tsai-Hill criterion were carried out. With this new setting, a maximum displacement of $1.91 \mathrm{~m}$ (mainly vertical deformation of the structure), and a maximum tensile stress of $235 \mathrm{MPa}$ in one of the sheets of Vectran (along the interface between rings where the horizontal ribs lie) have been detected. The structure assumes a shape based on a set of toroidal rings, whose section is circular, as expected with a uniform internal pressure (see Fig. 19).

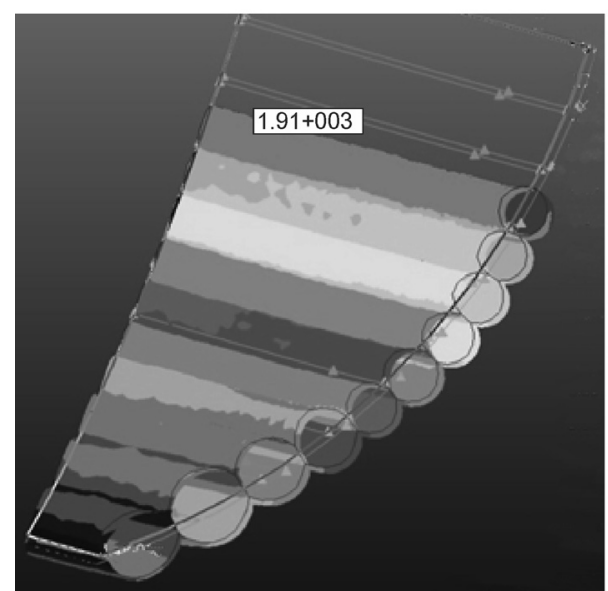

Fig. 19. Horizontal ribs final configuration FEM analysis with damping and fabric element: displacements in $\mathrm{mm}$

The final mass breakdown of the inflatable structure, excluding semi-spheres and cones was computed to be $315 \mathrm{~kg}$, divided into $145 \mathrm{~kg}$ for the external conical surface, $140 \mathrm{~kg}$ for the internal one, $30 \mathrm{~kg}$ for the ribs. The analyses have been carried out by increasing from 0 bar to 0.5 bar the internal pressure and continuing the simulation for 10 more seconds after the end of the inflation in order to let it completely relax.

\subsection{Semi-Spheres and Cones}

The final step of the analysis was to compute the stress over the semi-spheres and cones in order to size the thickness of its fabric. The cones and spheres are joined to the main structure, and eventually reinforcing strips can be adopted to increase the strength of the connection zone. After several FEM analyses, using a thickness of $0.5 \mathrm{~mm}$ and an internal pressure of 0.5 bar, the maximum tensile stress on the spheres and cones has been evaluated below $100 \mathrm{MPa}$ (see Fig. 20), with maximum displacement of $0.1 \mathrm{~m}$ in the top of the cones. The weight of the cones and spheres has been evaluated equal to $100 \mathrm{~kg}$, so that the mass of the inflatable part of the satellite structure is less than $420 \mathrm{~kg}$. Due to the uncertainties in the load modelling (impacts of the asteroids, local peaks of stress, junctions, fatigue), the high coefficient of safety obtained with Tsai-Hill criterion for 0.5 bar pressure load seems to be acceptable, at least for preliminarily analyses.

Such a weight of the satellite inflatable part (Fig. 21) is concurrent with the mass breakdown proposed by [1] and justifies further studies and experimental 
simulations to better detail the design of the inflatable basket.
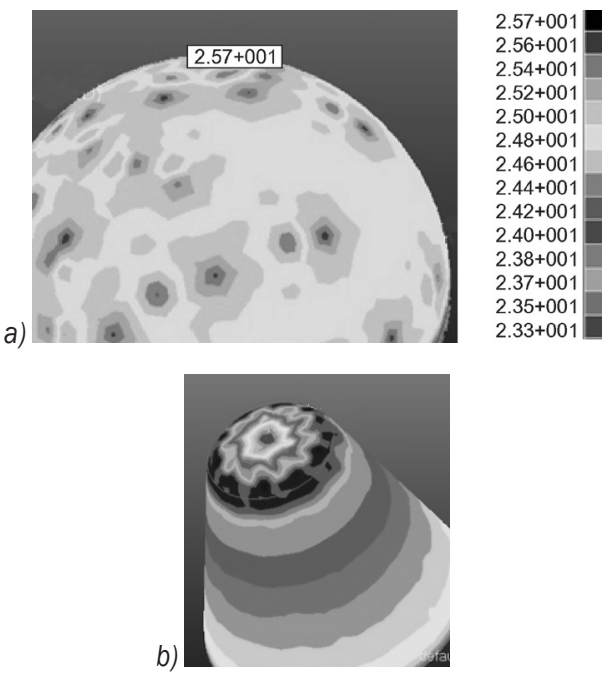

Fig. 20. Horizontal ribs configuration FEM analysis with damping and fabric element: a) sphere: displacements in $\mathrm{mm}$ and b) cones: stresses, colour map from light (13.6MPa) to dark (75.5 MPa)

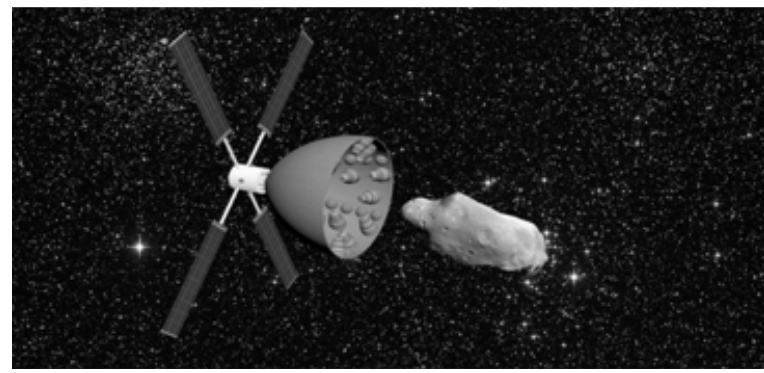

Fig. 21. Artistic impression of the authors of the inflatable structure and satellite in space

\section{RESULTS DISCUSSION AND CONCLUSION}

This paper presents a methodology to design, select, size and simulate, through FEM analyses, the inflatable section of a satellite whose mission is to capture an asteroid with a diameter of $7 \mathrm{~m}$. Damping should be applied to the FEM model to obtain the convergence of the displacements and stresses: in this study, the $\alpha$ damping is adopted and values for the $\alpha$ coefficient higher than what suggested by literature seem to improve the convergence. A fabric model keeping into account the compression behaviour of the fabric is necessary to simulate the weakness in compression and the following reduced stresses, so that a membrane element is suggested in FEM analyses. The final weight of the structure obtained after this study is in agreement with conceptual studies found in literature [1]. A further reduction in weight could be obtained with a better definition of the operative loads scenarios. The outcome of this study is the development of a methodology to rank possible configurations, the proposal of a shape for the inflatable basket, a list of guidelines to follow in FEM analysis of inflatable structures, and the definition of a feasible fabric thickness, valid for this case study. The use of inflatable structures for asteroids capturing presents weights compatible with the payload typical of a satellite, but further analysis and experimental tests to tune the FEM model are necessary. Further details, such as the modelling of the junction between fabric elements, and the simulation of impacts between asteroid and satellite should be better addressed in future works. Detailed trade-off studies to define the most suitable inflation pressure could be carried out once a real case-based simulation of asteroid capturing will be available in the relevant literature.

\section{ACKNOWLEDGMENTS}

Authors wish to thank Dr Giorgio Gaviraghi for suggesting this study and fruitful discussions and $\mathrm{Dr}$ Matteo Pettenuzzo for his support in the modelling of the configurations.

\section{REFERENCES}

[1] Asteroid Retrieval Feasibility Study (2012) prepared for Keck Institute For Space Studies, California Institute of Technology, Jet Propulsion Laboratory, Pasadena, available at: http:// www.kiss.caltech.edu/study/asteroid/asteroid_final_report. pdf, accessed, 2013-11-01.

[2] Brophy, J., Gershman, R., Landau, D., Polk, J., Porter, C., Yeomans, D., Allen, C., Williams, W., Asphaug, E. (2011). Asteroid Return Mission Feasibility Study, 47 th AIAA/ ASME/SAE/ASEE Joint Propulsion Conference \& Exhibit, American Institute of Aeronautics and Astronautics, Dol:10.2514/6.2011-5665.

[3] Stich, S. (2013). Asteroid Redirect Mission and Human Space Flight, Briefing to National Research Council Committee for Study on Human Space Flight (NASA-United States of America), available at http://www.nasa.gov/ pdf/756678main_20130619-NRC_Tech_Panel_Stich.pdf, accessed on 2014-01-28.

[4] Landis, R.R., Abell, P.A., Korsmeyer, D.J., Jones, T.D., Adamo, D.R. (2009). Piloted operations at near-Earth object (NEO), Acta Astronautica, vol. 65, no. 11-12 pp. 1689-1697, DOI:10.1016/j.actaastro.2009.04.022.

[5] Curtis, H. (2005). Orbital Mechanics for Engineering Students. Butterworth-Heinemann, Oxford.

[6] Cavallaro, P.V., Johnson, M.E. and Sadegh, A.M. (2003). Mechanics of plain-woven fabrics for inflated structures. 
Composite Structures, vol. 61, no. 4, p. 375-393, Dol:10.1016/ S0263-8223(03)00054-0.

[7] Apedo, K.L., Ronel, S., Jacquelin, E., Tiem, S. (2014). Free vibration analysis of inflatable beam made of orthotropic woven fabric. Thin-Walled Structures, vol. 78, p. 1-15, D0I:10.1016/j.tws.2013.12.004.

[8] Lachenmeier, K.S., Murai, K. (2004). Development of threedimensional reinforced membrane technology for high performance balloon and inflatable applications. Advances in Space Research, vol. 33, no. 10, p. 1732-1735, D0l:10.1016/j. asr.2003.07.045.

[9] Suhey, J.D., Kim, N.H., Niezrecki, C. (2005). Numerical modelling and design of inflatable structures: application to open-ocean-aquaculture cages. Aquacultural Engineering, vol. 33, no. 4, p. 285-303, D0l:10.1016/j.aquaeng.2005.03.001.

[10] Salama, M., Kou, C.P., Lou, M. (2000). Simulation of deployment dynamics of inflatable structures. AIAA Journal, vol. 38, no. 12 p. 2277-2283, Dol:10.2514/2.896.

[11] Lindell, M.C., Hughes, S.J., Dixon, M., Wiley, C.E. (2006). Structural analysis and testing of the inflatable re-entry vehicle experiment (IRVE). 47th $A I A A / A S M E / A S C E / A H S / A S C$ Structures, Structural Dynamics, and Materials Conference, AIAA-2006-1803, D0I:10.2514/6.2006-1699.

[12] Timmers, R., Hardy, R., Welch, J. (2009). Modeling and simulation of the second-generation Orion crew module airbag landing system. 20 th AIAA Aerodynamic Decelerator Systems Technology Conference and Seminar, D0I:10.2514/6.20092921.

[13] Stein, J., Sandy, C. (2003). Recent developments in inflatable airbag impact attenuation systems for Mars exploration. 44th AIAA/ASME/ASCE/AHS Structures, Structural Dynamics, and Materials Conference, AIAA-2003-1900, D0I:10.2514/6.20031900.
[14] Cadogan, D., Sandy, C., Grahne, M. (2002). Development and evaluation of the Mars pathfinder inflatable airbag landing system. Acta Astronautica, vol. 50, no. 10, p. 633-640, D0I:10.1016/S0094-5765(01)00215-6.

[15] Hinkle, J., Timmers, R., Dixit, A., Lin, J., Watson, J. (2009). Structural design, analysis, and testing of an expandable lunar habitat. 50th $A I A A / A S M E / A S C E / A H S / A S C$ Structures, Structural Dynamics, and Materials Conference, DOl:10.2514/6.2009-2166.

[16] Babuscia, A., Corbin, B., Knapp, M., Jensen-Clem, R., Van de Loo, M., Seager, S. (2013). Inflatable antenna for cubesats: Motivation for development and antenna design. Acta Astronautica, vol. 91, p. 322-332, D0l:10.1016/j. actaastro.2013.06.005.

[17] Lampani, L., Gaudenzi, P. (2010). Numerical simulation of the behavior of inflatable structures for space. Acta Astronautica, vol. 67, no. 3-4, p. 362-368, Dol:10.1016/j. actaastro.2010.02.006.

[18] Cui, D., Yan, S., Guo, X., Chu, F. (2012). An overview of dynamics modeling of inflatable solar array. Energy Procedia, vol. 14, p. 1967-1972, D0l:10.1016/j.egypro.2011.12.1195.

[19] Rao, R.V. (2007). Introduction to multiple attribute decisionmaking (MADM) methods - decision making in the manufacturing environment, Springer, London, p. 27-41.

[20] Ceruti, A., Marzocca, P. (2014). Conceptual approach to unconventional airship design and synthesis. Journal of Aerospace Engineering, vol. 27, no. 6, Dol:10.1061/(ASCE) AS.1943-5525.0000344.

[21] Joldes, G.R., Wittek, A., Miller, K. (2009). Computation of intra-operative brain shift using dynamic relaxation. Computer Methods in Applied Mechanics and Engineering, vol. 198, no. 41-44, p. 3313-3320, Dol:10.1016/j.cma.2009.06.012. 